\title{
Intelligent Car Anti-Theft Face Recognition System
}

\author{
https://doi.org/10.3991/ijoe.v17i01.18583 \\ V. Akila $\left.{ }^{\bowtie}\right)$, K. Sriharshini, P. Sravani, D. Sravanthi, Rishita Gopi \\ Gokaraju Rangaraju Institute of Engineering and Technology, Hyderabad, India \\ akila_beeyahoo.co.in \\ T. Sheela \\ Sri Sai Ram Engineering College, Chennai, India
}

\begin{abstract}
Security and safety of the car is the major problem of vehicle users in the present situation. The growth in Biometric Technologies has provided a way to solve the safety and security problems. Here, we use Open CV Face Detection Subsystem (FDS) which works using Global Positioning System (GPS) and Global System for Mobile Communication (GSM) module along with Arduino. This model provides a cheaper cost security system for four or more-wheeled vehicles. This project describes the foremost module for Open CV Face Detection Subsystem (FDS) which takes photos of driver using Open CV algorithm and compare it in database provided with photos of different drivers. The other modules are being useful to send all the authorized information about vehicle to the owner. The whole system works based on Arduino module. This system we can keep track of our vehicle in a very cheaper cost and more accurate. Using this system, the identification of the thief will be easy and tracking the location of the car will be faster and cheaper than earlier method.
\end{abstract}

Keywords - Global Positioning System (GPS), Global System for Mobile Communications (GSM), Open CV Face Detection System, Arduino

\section{Introduction}

The advancement of technology in different sectors has also brought many changes in advancement of Automobile Industry. Automobile companies have started their advancement in different technological aspects in hardware and enhanced their systems using software technology too. As far as vehicle security is concerned, there is no particular solution which has been used and implemented efficiently. The vehicle security system should be reliable, efficient and should be low cost. As per of research till date, GPS is the best way to keep track of vehicles. It traces the vehicle location and transfers it to desired user through GSM module. GSM module is used for keeping mobile interface with vehicle [1]. Sending SMS with specific template can perform different operations in vehicle. Security and commercial applications form the major improvement in showing for the past few years due to Automatic face 
recognition technologies. An automated-systems using Artificial Intelligence is real time task for new researchers in this origin [3].

As we are concerned to make vehicle security system with low cost, we use Arduino module as a controlling interface. Our system maintains the collection of user facial features as datasets and used them for verification. Many new technologies like image processing mechanism, biometric recognition, communication application and references have been combined into car security system [2]. We make use of face detection methods for car security system. The GPS module parse received signal to find accurate location. The GSM module is used to send SMS and images of the drivers to authorized users. All these processes are controlled under Arduino.

\section{Literature Survey}

Serial port is used to communicate between system and hardware components. Python also consists of different libraries like OpenCV, PCA algorithm which are used for face detection. Face detection by using Eigen value storage is easiest and accurate to work on.Eigen faces is one of the most investigated research for face recognition [4]. It is used in Open CV algorithm to efficiently represent pictures of faces. The face images could be rebuilt by adding of weights for each face icon and a face image. The weights for each face are obtained by extruding the face image onto the Eigen picture used Eigen faces, for face detection and identification [8].

The reference paper that we involved is Faces Recognition with Raspberry pi. There they used Raspberry pi which have different advantages like complex power supply, IO interfaces etc. [13]. IO interfaces are used to involve different types of pins which can be used for different sort of applications. There they have involved two pins for GSM and GPS module. Remaining all other pins can be used for other applications if needed. The raspberry phi module is advanced version where it also includes some features of Arduino [5]. The face recognition algorithm that they used is PCA algorithm. PCA Algorithm is the process of collecting different points around a surface which minimizes the distance between points. Repeating this process yields an orthogonal basis in which different dimensions of correlated data is collected and forms a vector which are called principal components and analysis is called principal component analysis. Those components are stored in database and checked for further validity. It also includes the message sending to both Arduino through GPS and GSM module [6].

The message includes the area where the vehicle is being and the image captured of the present vehicle user. The accuracy is up to the needed and working need less resources but need more pre-requisite is needed to operate the system. Eigen values are used for detecting the face edge values and make them to form a vector and store them in separate trainer module and used for mapping the detected face to identify the face features and map it [7]. 


\section{System Requirements}

\subsection{Hardware system configuration}

GSM module can send messages to phone number stored in database or to the server, which send SMS messages. GPRS module is used to send images of real time circumference in the car to the server. These modules are built inside the car security system [11]. GPS (Global Positional System) is another important module which plays an important role in intelligent vehicle development. It is used to send the location of the car to the desired user specified in the database.

Arduino can be used to take input from sensors and display output to lights, motors and other output devices. Arduino is used to give interaction between Face detection subsystem with GPS and GSM [9]. The simplified utilization of the I2C bus is by using the Arduino software. Arduino is used to make interaction between hardware and software [10]. It is the most powerful and flexible tool for experienced users. The quality information present in Arduino makes the inexperienced user to use Arduino in creating and exciting ways. The main aim of Arduinois is to make microcontrollers accessible to everyone with a minimal computer knowledge [12].

\subsection{Software system configurations}

Open CV detection algorithm is an open-source library in python used for face detection using Eigen values. The Eigen faces method took a holistic approach to face recognition. The basic idea is to maximize the variance between the classes and minimize the variance inner class at the same time[14]. Then the database is used for image recognition. It consists of a database which stores images based on Eigen values of each image. Whenever there is a need to retrieve or compare the stored image, it is by using Eigen values of each image. Eigen values are stored in database. Database consists of face datasets and is used to retrieve the data when needed [14].

\section{Software Environment}

Basically, face recognition algorithm works with python Interface. The system developed using Python (3.8version). We have divided the entire system into modules. For each module we wrote a separate script and run it under python platform. Arduino platform is used to send current location and address to the user and nearby police. When signal from python module creates a signal in serial port of Arduino interface. Thereafter, a sketch file which is already created with code is executed in which there will be code to send message to owner. Arduino is open source electronic platform where it acts as link between hardware and software. Arduino programming is done on Arduino platform (IDE). 


\section{$5 \quad$ Face Recognition Algorithm}

PCA Algorithm is carried out in two phases. The first phase is called training phase and second phase is called testing phase. In the training phase, the face images of the authenticated driver are stored in the database and weight vector is calculated for each image. In the testing phase, the test image weight vector is calculated and compared with the threshold value of training image. If the value is below the threshold value then image is authenticated otherwise send the image of the driver and location of the car to the owner. If the owner identified as unauthorized image then the image and the location of car is transferred to the nearest police station.

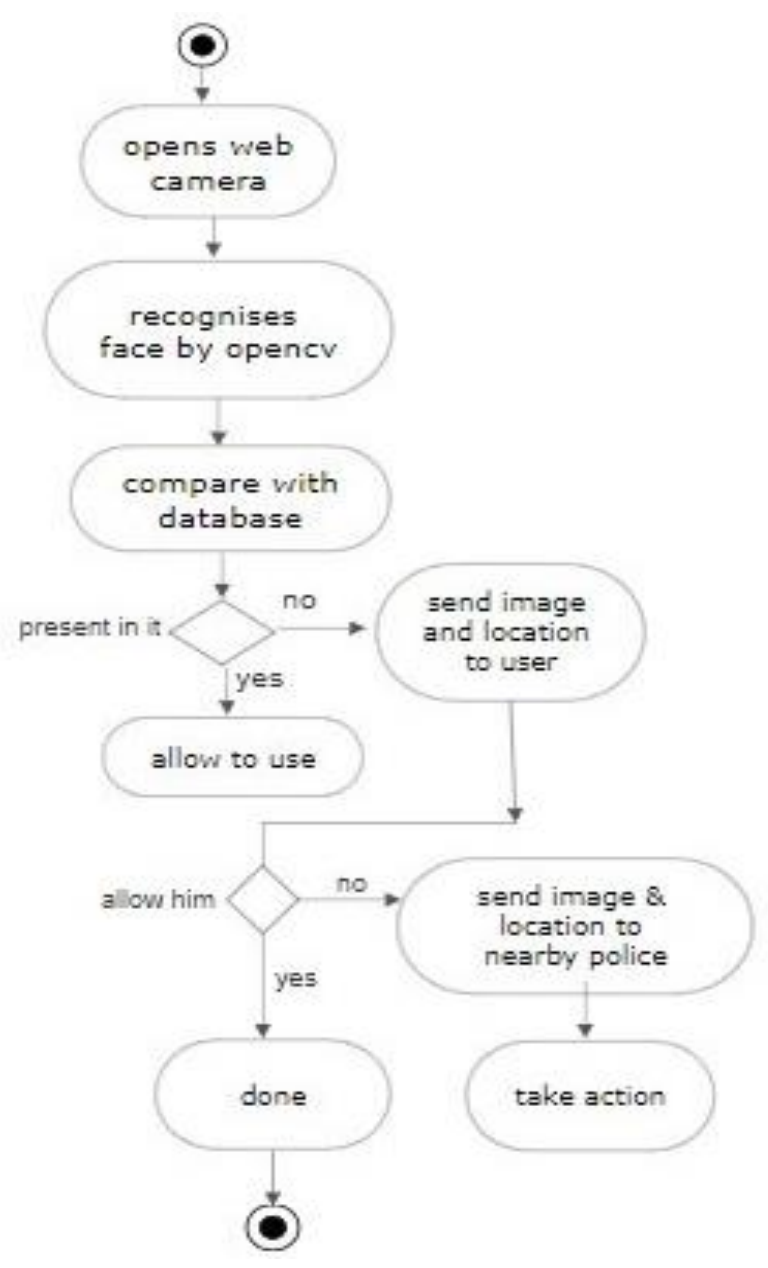

Fig. 1. Algorithm for Face Recognition

The procedure followed in PCA algorithm is specified in Fig.1. The following are the steps to be performed in PCA Algorithm. 
Step 1: Divide the set of images into training and testing images.

Step 2: Input the training images and enter into face recognition stage.

Step 3: Calculate the weight vector for training images using PCA Algorithm.

Step 4: Input the test images.

Step 5: Check test images are authenticated using PCA Algorithm.

Step 6: If authentication failed then the image of unauthenticated user and location of car send to the owner.

Step 7: If the owner identified the image as unauthorized then image and the location of the car details send to the nearby police station.

\section{Implementation}

\subsection{Framework needed for time constraints}

The project is efficient to work and the result is accurate. It can be useful even in 6 and 8 wheelers vehicles. It can even provide secured encryption for the vehicle by providing invalid information to nearby police. It also provides the current location of vehicle using GPS tracking.

\subsection{System architecture}

The system consists of Arduino board, GPS module and GSM module. Arduino board is connected to system using USB cable. Serial port communication is made through changing the mode to Arduino UNO in tools. We connect GSM module to Arduino board by connecting pin 3 of Arduino to the RX pin of the GPS Module and also connect pin 4 of Arduino to the TX pin of the GPS Module. We connect GPS module to Arduino board by checking the power requirements of module and verifying the title output pins in the module. The steps to add mobile interface through GSM module are insert the SIM card to GSM module and lock it, link the adapter to GSM module and turn it on, now wait for sometimes until blink of led and once connection established, the network led blink for every 3 seconds. Arduino consist of library called Serial port where it provides interface to software and hardware. Once all these are connected, we have established connection between hardware and software. Then we make code to run.

\section{$7 \quad$ Result and Discussions}

First images are identified as different copies for each and features are being identified and stored in a file. The result of the system consists of the image folder in which all trained images is stored using OpenCV algorithm. Each copy of image has a different feature. Image contain different features and all the feature set of all images are stored in trainer file present in folder which have identified images which is called image database set. In this we use only OpenCV and Numpy library to work 
on. When we track the images, if it is not present it's got track and stores the image in folder which contain unauthorized images as shown in Fig.2.

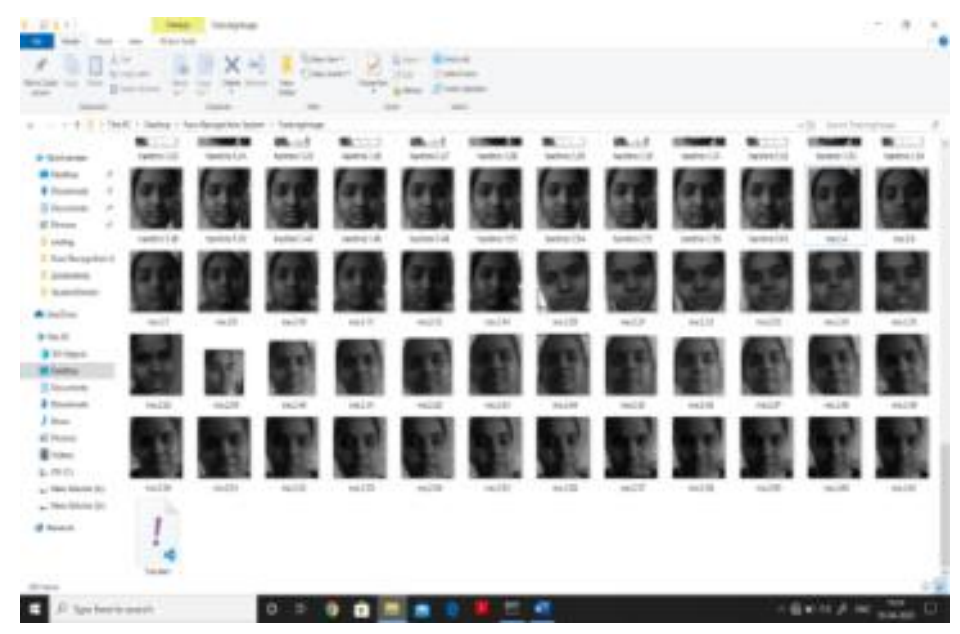

Fig. 2. Shows Authorized Images and its Features

When known image is identified the code allows accessing the vehicle. If the image is not present, the camera identifies it as unknown and stores it in a different folder. Even also the unknown images around are stored as shown in Fig.3.

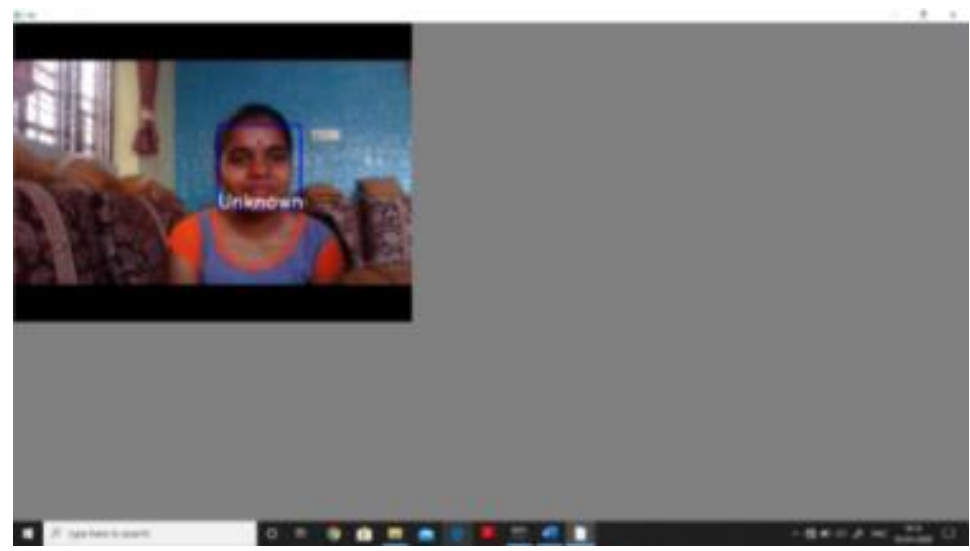

Fig. 3. Shows the Authorized Detected Image

The unknown image is stored in different folder and serial counter message is sent to Arduino in order to send message to owner and share location. If the counter sent is 0 image is present in saved folder. If the counter sent is 1 , image is not present and makes to send message to owner and nearby police. The serial code is used to send code for connecting Arduino and Face Recognition Algorithm. The code sends signal to Arduino. Arduino will send message to owner and nearby police. The code for 
GSM and GPS are used for connection between the two devices. The final message sent to the owner of the vehicle with given phone number in the database. The output of the system will be notified along with location in the message.

\section{Conclusion}

The system presented a start for the automatic recognition and detection of individual by their face as input mode. We have involved this because of great demand for such a system by security services, agencies, investigative services etc. The system worked very well and it overcame certain obstacles by using some innovative techniques. The system uses a multimodal approaches to improve the recognition rates and used face as input mode. The face combines to improve the recognition rates as was shown in the experimental section over the same metrics used in an all other methods. One achievement of the system, is that the multimodal biometric system presented here is totally automated, requiring no manual intervention. Automated biometric recognition system can be mainly used in installations requiring person recognition using their face. The research displays the possibilities for a system of detection and recognition of individuals without their full cooperation. Another advantage of face a multimodal system is that they can compensate for one another, such as if one modality is occluded (e.g. hair, sun glasses etc.) a biometric can be achieved by using the other modality. As the images are being stored in separate folder, we can also keep track of vehicle thieves.

\section{$9 \quad$ Future Works}

The present project and research have been motivated by the need for a person identification system which is accurate. The face recognition system works well for image segmentation by employing both colour and depth information. While the system achieved results from experimentation, from these also came new ideas and areas which could be explored in some future research and work. A major change has been noticed in the recognition values using pre-processing and different feature extraction methods. Specially before cropping an image while it is run through a recognition algorithm, there must be much more to get through this segment. It would be attractive to examine new techniques of pre-processing that would drive to the optimal recognition vector values. In this case, face biometrics has been used, but perhaps there are other metrics that can be combined that will lead to a more robust and efficient system. The system provides the template security for the biometric fuzzy vault database, but still it can be investigate for the large-scale operation and integrating another biometric with the face and feature set. 


\section{References}

[1] H.K. Ekenel, R. Stiefelhagen, "Analysis of Local Appearance-based Face Recognition on FRGC 2.0 Database”, Face Recognition Grand Challenge Workshop (FRGC), Arlington, VA, USA, March 2006. https://doi.org/10.7717/peerjcs.236/fig-2

[2] H.K. Ekenel, R. Stiefelhagen," Analysis of local appearance-based face recognition: Effects of feature selection and feature normalization", Conference: Computer Vision and Pattern Recognition Workshop, 2006. https://doi.org/10.1109/cvprw.2006.29

[3] Qingcai Huang, Donghong Li, Qingyun Huang, “A bi-direction guard system design of car based on GPS and GSM", International Conference on Computational Aspects of Social Networks,2010. https://doi.org/10.1109/cason.2010.119

[4] JunLi, "A Novel Remote Sensing Image Classification Algorithm based on PCA and Hidden Marlkov Random Field Theory”, International Conference on Green Engineering and Technologies,2016. https://doi.org/10.1109/get.2016.7916783

[5] Chaohui Lü, Xi Wang, Yinghua Shen," A Stereo Vision Measurement System Based on OpenCV", 6th International Congress on Image and Signal Processing,2013. https://doi.org/10.1109/get.2016.7916783

[6] M.Malathi, R.Sujitha, M.R.Revathy, "Alcohol Detection and Seat Belt Control System using Arduino", International Conference on Innovations in information Embedded and Communication Systems, 2017. https://doi.org/10.1109/iciiecs.2017.8275841

[7] Vinoth Kumar Sadagopan, Upendran Rajendran, Albert Joe Francis, "Anti_Theft Control System Design Using Embedded System", IEEE International Conference on Vehicular Electronics and Safety,2011. https://doi.org/10.1109/icves.2011.598 $\underline{3776}$

[8] Frazer K. Noble," Comparison of OpenCV's Feature Detectors and Feature Matchers", 23rd International Conference on Mechatronics and Machine Vision in Practice (M2VIP), 2016 https://doi.org/10.1109/m2vip.2016.7827292

[9] A. S. Tolba, A.H. El-Baz, and A.A. El-Harby" Face Recognition: A Literature Review", International Journal of Signal Processing,2016.

[10] V.Akila, T.Sheela, "Preserving Data and Key Privacy in Data Aggregation for Wireless Sensor Networks," in IEEE second International Conference on Computing and Communications Technologies on 24th Feb 2017. https://doi.org/10.1109/iccct2.2017. 7972286

[11] V.Akila, T.Sheela, "Efficient Packet Scheduling Technique for Data Merging in Wireless Sensor Networks" in the China Communications (Web of Science, SCIE and IEEE indexed ) in Volume 14 ,Issue No. 4,Page No: 35-46, April 2017. https://doi.org/10. 1109/cc.2017.7927575

[12] Aisha Y Alsobhi, Khaled H Alyoubi," Learning Styles and Dyslexia Types Understanding Their Relationship and its Benefits in Adaptive E-learning Systems", International Journal of Interactive Mobile Technologies (iJIM), January 2020. https://doi.org/10.3991/ijim.v14i15.16129

[13] Hanif Fakhrurroja, Carmadi Machbub, Ary Setijadi Prihatmanto, Ayu Purwarianti," Multimodal Interaction System for Home Appliances Control”, International Journal of Interactive Mobile Technologies (iJIM), January 2020. https://doi.org/10.3991/ijim. v14i15.13563

[14] Ghazanfar Ali Abbasi, Sea Su-Yee, Yen-Nee Goh, “An Integrative Approach for Determining Consumers Mobile Advertising Related Attitudes and Intentions", International Journal of Interactive Mobile Technologies (iJIM),January 2020 https://doi.org/10.3991/ijim.v14i15.14955 


\section{Authors}

Dr. V. Akila, is working as Associate Professor, Department of Information Technology in Gokaraju Rangaraju Institute of Engineering and Technology. She had presented papers in national and international conferences and published papers in international journals. Her research interests include Wireless and Mobile sensor networks, Artificial Intelligence, and Internet of Things. Email: akila_be@ yahoo.co.in

K. Sriharshini, Rishita Gopi, D. Sravanthi and P. Sravani are studying B. Tech final year students in Gokaraju Rangaraju Institute of Engineering and Technology in the stream of Information Technology.

Dr. T. Sheela, is working as a Professor and head, Department of Information Technology at Sri Sai Ram Engineering College for the past 15 years. She has been in academics for the past twenty-five years. Her research area is "Network Protocols". She had presented around 30 papers in the related topic in national and international conferences and more than 15 research publications in reputed journals.

Article submitted 2020-09-15. Resubmitted 2020-11-16. Final acceptance 2020-11-22. Final version published as submitted by the authors. 